\title{
Gender-Specific Effects of Prenatal and Adolescent Exposure to Tobacco Smoke on Auditory and Visual Attention
}

\author{
Leslie K Jacobsen*, ${ }^{*, 2,3}$, Theodore A Slotkin ${ }^{4}$, W Einar Mencl ${ }^{3}$, Stephen J Frost ${ }^{3}$ and Kenneth R Pugh ${ }^{2,3}$ \\ 'Department of Psychiatry, Yale University School of Medicine, New Haven, CT, USA; ${ }^{2}$ Department of Pediatrics, Yale University School \\ of Medicine, New Haven, CT, USA; ${ }^{3}$ Haskins Laboratories, New Haven, CT, USA; ${ }^{4}$ Department of Pharmacology and Cancer Biology, \\ Duke University Medical Center, Durham, NC, USA
}

\begin{abstract}
Prenatal exposure to active maternal tobacco smoking elevates risk of cognitive and auditory processing deficits, and of smoking in offspring. Recent preclinical work has demonstrated a sex-specific pattern of reduction in cortical cholinergic markers following prenatal, adolescent, or combined prenatal and adolescent exposure to nicotine, the primary psychoactive component of tobacco smoke. Given the importance of cortical cholinergic neurotransmission to attentional function, we examined auditory and visual selective and divided attention in 181 male and female adolescent smokers and nonsmokers with and without prenatal exposure to maternal smoking. Groups did not differ in age, educational attainment, symptoms of inattention, or years of parent education. A subset of 63 subjects also underwent functional magnetic resonance imaging while performing an auditory and visual selective and divided attention task. Among females, exposure to tobacco smoke during prenatal or adolescent development was associated with reductions in auditory and visual attention performance accuracy that were greatest in female smokers with prenatal exposure (combined exposure). Among males, combined exposure was associated with marked deficits in auditory attention, suggesting greater vulnerability of neurocircuitry supporting auditory attention to insult stemming from developmental exposure to tobacco smoke in males. Activation of brain regions that support auditory attention was greater in adolescents with prenatal or adolescent exposure to tobacco smoke relative to adolescents with neither prenatal nor adolescent exposure to tobacco smoke. These findings extend earlier preclinical work and suggest that, in humans, prenatal and adolescent exposure to nicotine exerts gender-specific deleterious effects on auditory and visual attention, with concomitant alterations in the efficiency of neurocircuitry supporting auditory attention.
\end{abstract}

Neuropsychopharmacology (2007) 32, 2453-2464; doi:I0.1038/sj.npp. I 30I398; published online 2I March 2007

Keywords: adolescence; nicotine; brain development; maternal smoking; auditory attention; visual attention

\section{INTRODUCTION}

Controlled, prospective clinical studies have linked maternal smoking during pregnancy with persistent deficits in general intellectual function and auditory processing in offspring (Fried et al, 1997, 2003; McCartney et al, 1994). Converging evidence from preclinical work has identified nicotine as the chief teratogenic component of tobacco smoke contributing to adverse neurodevelopmental sequelae associated with prenatal exposure to maternal smoking (Slotkin, 2004; Slotkin et al, 2007). Nicotine binds to nicotinic acetylcholine receptors (nAChRs) which, when stimulated by endogenous acetylcholine, play a key role in the regulation of brain development, promoting cell replication, differentiation, and synaptic development

*Correspondence: Dr LK Jacobsen, Psychiatry and Pediatrics, Yale University School of Medicine, 2 Church Street South, Suite 207, New Haven, CT 06519, USA, Tel: + I 203764 8480, Fax: + I 203 764 8484, E-mail: leslie.jacobsen@yale.edu

Received 20 December 2006; revised I4 February 2007; accepted 16 February 2007
(Lauder and Schambra, 1999; Metherate and Hsieh, 2004; Slotkin, 2004). Stimulation of nAChRs by nicotine during prenatal development produces persistent cholinergic and serotonergic hypoactivity, and reductions in neural cell membrane complexity, alterations in neurotransmitter responses mediated through adenyl cyclase and alterations in serotonin receptor expression (Navarro et al, 1989; Slotkin, 2004; Slotkin et al, 2007). The vulnerability of the developing brain to nicotine extends into the early postnatal period in the rodent (corresponding to the third trimester in humans), where exposure disrupts auditory learning and nicotinic regulation of primary auditory cortex (Liang et al, 2006).

Neurodevelopment continues through adolescence, with ongoing frontal, parietal, and perihippocampal myelination and reductions in cortical synaptic density (Benes et al, 1994; Giedd et al, 2006; Huttenlocher, 1979; Lenroot and Giedd, 2006; Yakovlev and Lecours, 1967). Work in rodents has provided evidence that nicotine is also disruptive to adolescent brain development, resulting in changes in cholinergic neurotransmission, cell membrane complexity, cell signaling, and serotonin receptor density that are 
similar to but smaller in magnitude than those observed following prenatal nicotine exposure (Abreu-Villaca et al, 2003a, b; Slotkin et al, 2007). Consistent with this evidence that vulnerability to the developmentally disruptive effects of nicotine extends into adolescence, adolescent tobacco smokers were found to have impaired working memory performance accuracy, while male smokers demonstrated impairments of auditory selective attention relative to female smokers and to male and female nonsmokers (Jacobsen et al, 2005). These effects were observed regardless of how recently subjects had smoked, and thus could not be ascribed to acute effects of nicotine withdrawal on cognition (Jacobsen et al, 2005). Evidence from a longitudinal sample studied before and after adolescent initiation of smoking has shown that smoking-associated cognitive deficits persist after controlling for presmoking levels of cognitive performance, further suggesting that cognitive deficits observed in adolescent smokers may stem from smoking (Fried et al, 2006).

Rates of tobacco smoking and nicotine dependence are elevated among offspring prenatally exposed to maternal smoking (Buka et al, 2003; Cornelius et al, 2000; Griesler et al, 1998; Kandel et al, 1994). Recent work with a rodent model developed to study the effects of combined nicotine exposure during these two key developmental epochs (gestation and adolescence) has provided evidence of sexspecific effects (Slotkin et al, 2007). Whereas effects of prenatal or adolescent nicotine exposure alone on cholinergic neurotransmission, neural cell membrane complexity, cell signaling, and serotonin receptor expression were more prominent in males than in females, when prenatal exposure was followed by adolescent exposure the net effect on indices of neurodevelopment in females was similar to that in males (Slotkin et al, 2007). In contrast, among males, the magnitude of effects of dual (prenatal + adolescent) nicotine exposure on neurodevelopment was similar to that observed with prenatal or adolescent exposure alone (Slotkin et al, 2007).

A large body of evidence (reviewed in Sarter et al, 2005) has shown that normal attentional performance rests on the integrity of cortical cholinergic neurotransmission. Although exposure to nicotine during brain development has been shown to reduce cortical cholinergic markers in the rodent model (Slotkin et al, 2007), few prior studies have examined the impact of prenatal and adolescent exposure to tobacco smoke on attention in humans. Accordingly, we examined auditory and visual attention in 181 adolescent smokers and nonsmokers with and without prenatal exposure to active maternal smoking. Given recent preclinical work demonstrating sex differences in the pattern of reductions in cholinergic markers resulting from nicotine exposure during prenatal and adolescent development (Slotkin et al, 2007), we anticipated that although females would exhibit greater deficits following combined prenatal and adolescent exposure to tobacco smoke than following prenatal or adolescent exposure alone, males would exhibit a similar magnitude of deficits following prenatal or adolescent exposure alone or in combination. Given earlier clinical work demonstrating greater deficits in auditory attention in male adolescent smokers relative to male nonsmokers and to females (Jacobsen et al, 2005), we anticipated that deleterious effects of exposure to tobacco smoke during brain development on attention would be greater in the auditory modality in males. A subset of subjects was studied using functional magnetic resonance imaging (fMRI) during performance of a task assessing auditory and visual attention. Earlier work has shown that increases in cortical cholinergic neurotransmission enhance the selectivity of perceptual processing (Furey et al, 2000; McGaughy et al, 1996; McGaughy and Sarter, 1998; Sarter et al, 2005), and improve the efficiency (reduce activation) of brain regions supporting higher cortical function (Furey et al, 2000). Thus, we anticipated that exposure to tobacco smoke during brain development would be associated with reduced efficiency (increased activation) of brain regions supporting attentional processing, possibly reflecting nicotine exposure-induced reductions in cortical cholinergic neurotransmission.

\section{METHODS}

\section{Participants}

Ninety-two adolescents with prenatal exposure to active maternal smoking were compared to 89 adolescents with no prenatal exposure to active maternal smoking. Prenatally exposed subjects included 67 current daily tobacco smokers and 25 nonsmokers (defined as having a lifetime history of smoking no more than two cigarettes), whereas subjects with no prenatal exposure to maternal smoking included 44 current daily tobacco smokers and 45 nonsmokers. Participants were 13 to 18 years of age and were recruited from the community by advertisement. All subjects were free of medical and DSM IV (Diagnostic and Statistical Manual of Mental Disorders, Fourth Edition; American Psychiatric Association, 1994) psychiatric illness and substance abuse or dependence disorders, other than nicotine dependence, as determined by physical examination and structured clinical interview (Kaufman et al, 1997; Meyers et al, 1995; Sobell and Sobell, 1992). Detailed druguse history was obtained using the Comprehensive Addiction Severity Index for Adolescents and the Time Line Follow-back Interview (Meyers et al, 1995; Sobell and Sobell, 1992). Abstinence from substance use during the week before assessment was confirmed by urine toxicology screen.

At initial screening, general intelligence was estimated using the Kaufman Brief Intelligence Test (Bowers and Pantle, 1998), reading achievement was estimated using the Word Attack Subtest of the Woodcock-Johnson Revised Test of Achievement, and self-reported symptoms of depression and inattention were assessed using the Beck Depression Scale (Beck et al, 1961) and the Conners Adolescent Self Report Scale (Conners, 1998), respectively. Prenatal exposure was assessed by semi-structured interview of the parents regarding the average number of cigarettes smoked per day by the mother during each trimester of her pregnancy with the subject. The mother's consumption of other substances and alcohol during the pregnancy, and the rate of smoking by other persons living in the home during the pregnancy (prenatal environmental exposure) were also assessed during this interview. 
Parental consent was obtained for subjects 17 years of age and younger. This study was approved by the Yale University School of Medicine Human Investigation Committee. Subjects provided written assent or, for 18-year olds, consent for study participation.

\section{Procedure}

In adults and adolescent humans, cessation of regular tobacco smoking produces an acute withdrawal syndrome, one characteristic of which is disruption of attention and memory (Jacobsen et al, 2005; Pineda et al, 1998; Shiffman et al, 1995; Snyder et al, 1989; West and Hack, 1991). To minimize potentially confounding effects of nicotine withdrawal on cognitive function, smokers were permitted to smoke their own brand of cigarettes during a break midway through behavioral testing and immediately before scanning. All smokers smoked during these breaks. During assessment, blood or saliva in 110 subjects was obtained to measure cotinine, the primary metabolite of nicotine. The proportion of subjects declining to provide blood did not significantly differ across groups. Saliva and plasma samples were frozen at $-30^{\circ} \mathrm{C}$ immediately following collection, and were analyzed using gas chromatography (Jacob et al, 1981). Self-reported mood was assessed using the Center for Epidemiologic Studies-Depression (CES-D) scale (Radloff, 1977). Among smokers, measures of symptoms of nicotine withdrawal, using the Minnesota Nicotine Withdrawal Scale (Hughes and Hatsukami, 1998) and the Clinical Institute for Narcotic Withdrawal Scale (Peachey and Lei, 1988), and tobacco craving, using the Shiffman Jarvik scale (1796), were also obtained at the time of assessment.

\section{Assessment of Auditory and Visual Selective and Divided Attention}

Subjects performed a modified version of a previously described computerized word recognition task that involved three progressively more demanding manipulations of attention (Shafritz et al, 2004; Shaywitz et al, 2001). Trials within each task were $5 \mathrm{~s}$ long. In the selective attention task, trials began with a $500 \mathrm{~ms}$ visual cue, depicting an eye or an ear, prompting subjects to attend to the visual or auditory modality, respectively (Figure 1a and b). After a $500 \mathrm{~ms}$ pause, a word or nonword was presented in the cued modality. Subjects then made a word/nonword discrimination (lexical decision) with a button press. In the simple condition, word or nonword stimuli were presented only in the attended modality, whereas nonlinguistic stimuli (four diagonal lines or a tone stimulus) were presented in the unattended modality. In the selective attention condition, a linguistic stimulus (word or nonword) was simultaneously presented in the unattended modality, thereby placing greater demand on neurocircuits mediating perceptual selectivity. In the divided attention task, word or nonword linguistic stimuli were presented in both modalities simultaneously (Figure 1c). After a $500 \mathrm{~ms}$ pause, a cue depicting an eye and an ear, representing the visual and the auditory modality, with a circle around the eye, the ear, both, or neither, was presented. Subjects responded positively with a button press if the stimulus presented in

a Visual Selective Attention Task

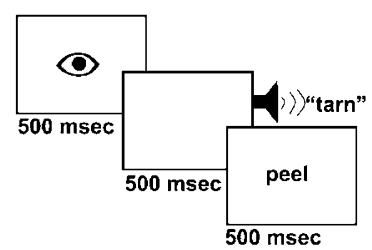

b Auditory Selective Attention Task

西

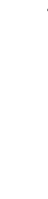




\section{Data Analysis}

Accuracy of task performance was assessed by dividing the number of correct responses by the total number of trials (proportion correct) for each condition. Data were analyzed using linear mixed effects regression analysis implemented in SPlus (Insightful Coorporation, Seattle, WA, USA) with subject modeled as a random effect. Models used to assess speed and accuracy of divided attention task performance included fixed effects for prenatal exposure (exposed $v s$ no prenatal exposure), smoking status (smoker/nonsmoker), gender, and the interactions between prenatal exposure, smoking status, and gender. Models used to assess speed and accuracy of selective attention task performance also included fixed effects for modality (auditory/visual) and selective attention load (simple/select). Potential confounding effects of baseline symptoms of depression (Beck score), estimated full scale IQ, reading achievement, alcohol use, lifetime episodes of cannabis use, prenatal exposure to environmental tobacco smoke, and prenatal exposure to maternal alcohol consumption were controlled by entering these variables as fixed effects in the regression models. Previous work has shown that salivary concentrations of cotinine in youth are, on average, $20 \%$ higher than plasma concentrations of cotinine (Jarvis et al, 2003). Therefore, salivary cotinine concentrations were multiplied by a factor of 0.8 before combining them with measures of cotinine from plasma.

Image analysis was performed using locally developed software written in Matlab (MathWorks, Natick, MA). Before statistical analysis, images were motion-corrected using SPM 99, and were spatially filtered using a Gaussian filter of full width at half maximum of $6.25 \mathrm{~mm}$. One volume was discarded at the end and at the beginning of each block to avoid variance stemming from hemodynamic changes that occur during task transitions. Single subject activation data was obtained using linear regression to generate maps of contrast values (regression parameter estimates) by comparing the mean signal during each activation condition to the control condition. Predictors for nuisance variables were also included in the regression model to account for run-to-run differences in mean signal intensity, and first-, second-, and third-order polynomial time trends (drift) within each run.

Before across subjects comparisons, fMRI data were spatially normalized to standard stereotactic space (Montreal Neurologic Institute Template) using BioImage Suite (www.bioimagesuite.org; New Haven, CT), as described previously (Ashburner and Friston, 1999; Papademetris et al, 2003). Effects of prenatal exposure to maternal smoking, adolescent smoking status, selective attention load, and modality on task-related activation were assessed at each voxel using mixed model repeated measures analysis of variance (Woods, 1996), with prenatal exposure and smoking status as between-subjects variables, modality and attention load as within-subjects variables, and subject treated as a random effect within each group. A univariate voxel threshold of $p<0.0001$, corrected for mapwise false discovery rate, (Genovese et al, 2002) was used with a further cluster threshold of ten contiguous significant voxels. Location of peak differences in activation were estimated from Talairach and Tournoux (Talairach and
Tournoux, 1988), after adjustment for differences between MNI and Talairach coordinate space using the nonlinear transformation by Brett (www.mrc-cbu.cam.ac.uk/Imaging/ Common/mnispace.shtml).

\section{RESULTS}

Demographic, clinical, and cognitive characteristics of the entire sample are presented in Table 1.

The groups did not differ in age, symptoms of inattention, years of education, or years of parent education. Smokers had significantly more symptoms of depression at screening $(\beta=4.7, t=2.9, p<0.01)$, greater history of alcohol $(\beta=2.4$, $t=2.3, p<0.05)$, and cannabis $(\beta=210.2, t=3.0, p<0.01)$ consumption, and lower estimated general intelligence $(\beta=-7.1, t=-2.5, p<0.05)$. These effects of adolescent smoking were not significantly modified by gender or prenatal exposure to maternal smoking. Birth weight of adolescents with prenatal exposure to maternal smoking was, on average, $0.26 \mathrm{~kg}$ lower than that of adolescents with no prenatal exposure to maternal smoking $(\beta=-7.5$, $t=-2.4, p<0.05)$. In addition, adolescents with prenatal exposure to maternal smoking had more environmental tobacco smoke exposure during gestation (persons other than the mother smoking in the home during gestation of the subject; $\beta=23.1, t=3.4, p<0.001)$. These effects of prenatal exposure were not significantly modified by gender or smoking status.

Among smokers, effects of gender and prenatal exposure to maternal smoking on estimated plasma cotinine and nicotine concentrations, age at onset of smoking, and symptoms of nicotine withdrawal and tobacco craving at assessment were not significant. Urine toxicology screen before assessment was positive for 11-nor- $\Delta^{9}$-tetrahydrocannabinol-9-carboxylic acid in eight smokers with prenatal exposure to maternal smoking and in 13 smokers with no prenatal exposure to maternal smoking, indicating the presence of residual cannabinoids. Urine toxicology screen was negative for all other substances of abuse in these subjects and was negative for all substances in all other subjects.

Among the adolescents with prenatal exposure to maternal smoking, exposure was restricted to the first trimester in 36, whereas five were exposed through two trimesters, and 51 were exposed throughout gestation. Reported maternal use of other drugs during pregnancy was as follows: among mothers of smokers with prenatal exposure to maternal smoking, 14 consumed alcohol, six consumed cannabis, and one consumed cocaine during the index pregnancy. Among mothers of smokers without prenatal exposure to maternal smoking, three consumed alcohol, one consumed cannabis, and one consumed cocaine during the pregnancy. Among mothers of nonsmokers with prenatal exposure to maternal smoking, six consumed alcohol, one consumed cannabis, and one consumed cocaine during the pregnancy. Among mothers of nonsmokers without prenatal exposure to maternal smoking, two consumed alcohol and none consumed cannabis or cocaine during the pregnancy. No subject in the sample was exposed to opiates, amphetamine, hallucinogens, sedative-hypnotics, or inhalants during gestation. 
Table I Demographic, Clinical and Cognitive Characteristics of 92 Adolescents with and 89 Adolescents without Prenatal Exposure to Active Maternal Smoking ${ }^{\mathrm{a}}$

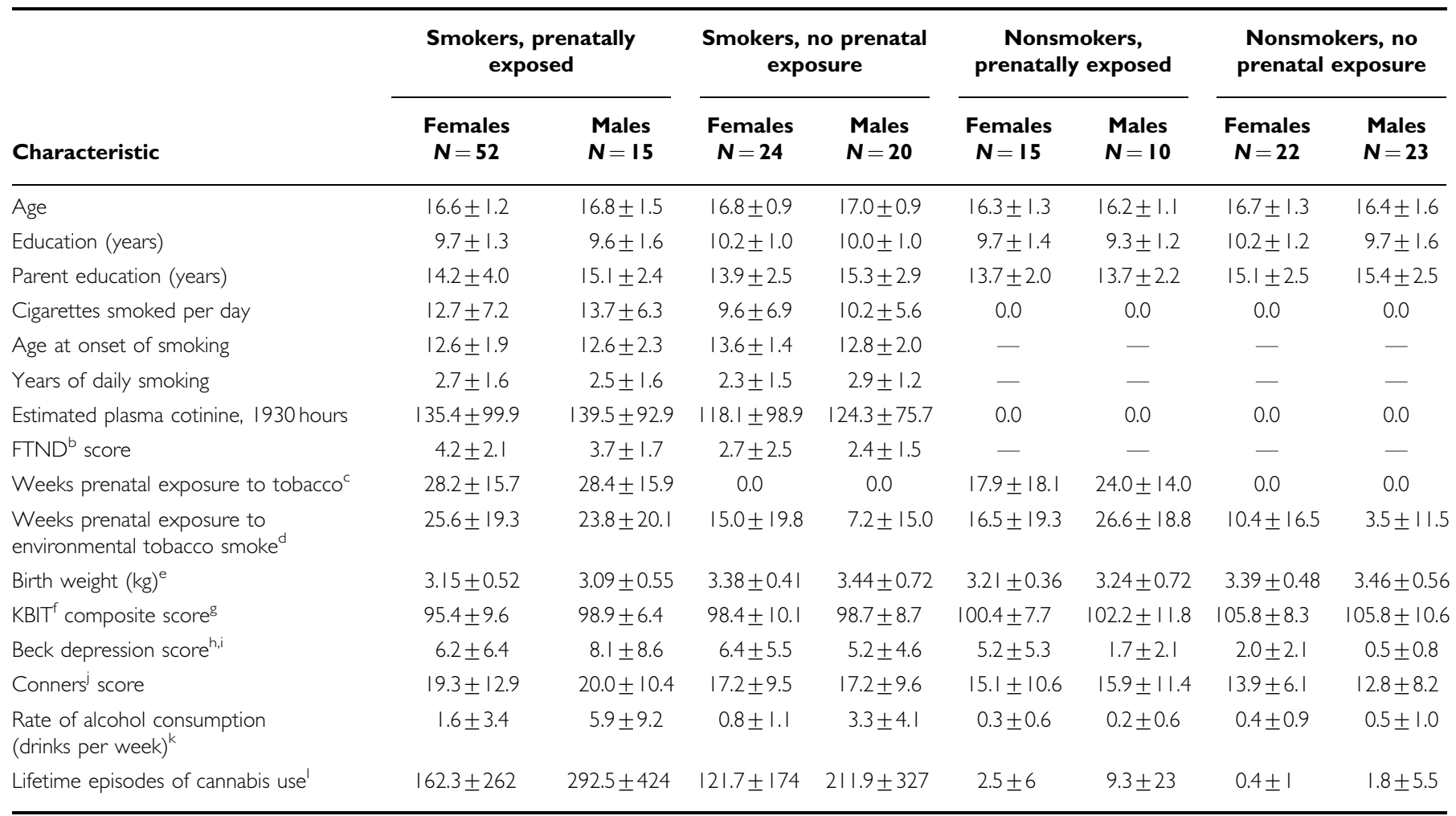

${ }^{a}$ Data are presented as mean \pm standard deviation, unless otherwise specified.

${ }^{b}$ Fagerstrom Test for Nicotine Dependence (Heatherton et al, 1991).

'Prenatally exposed $>$ no prenatal exposure: $\beta=24.0, t=5.5, p<0.0000$ I

dPrenatally exposed $>$ no prenatal exposure: $\beta=23.1, t=3.4, p<0.001$.

ePrenatally exposed $<$ no prenatal exposure: $\beta=-7.5, t=-2.4, p<0.05$.

${ }^{f}$ Kaufman Brief Intelligence Test (Bowers and Pantle, 1998).

'Smokers < nonsmokers: $\beta=-7.1, t=-2.1, p<0.05$.

h(Beck et al, 1961).

'Smokers $>$ nonsmokers: $\beta=4.7, t=2.9, p<0.01$.

${ }^{\mathrm{j}}$ Conners Adolescent Self Report Scale (Conners, 1998).

${ }^{k}$ Smokers $>$ nonsmokers: $\beta=2.4, t=2.3, p<0.05$.

'Smokers $>$ nonsmokers: $\beta=210.2, t=3.0, p<0.01$.

Adolescents with prenatal exposure to active maternal smoking also had more prenatal exposure to alcohol $(\beta=0.4, t=3.0, p<0.01)$. This effect was not significantly modified by gender or smoking status. Rates of prenatal exposure to cannabis or cocaine did not significantly differ across the groups.

Seven smokers with prenatal exposure to maternal smoking had experimented with illicit substances other than cannabis; four had tried cocaine, one had tried amphetamine, four had tried oral opiates, three had tried sedative-hypnotics, and five had tried hallucinogens. Six smokers with no prenatal exposure to maternal smoking had experimented with illicit substances other than cannabis; four had tried cocaine, one had tried amphetamine, three had tried oral opiates, two had tried sedativehypnotics, and one had tried hallucinogens. Nonsmokers with and without prenatal exposure to maternal smoking denied previous use of cocaine, amphetamine, opiates, sedative hypnotics, and hallucinogens, and all subjects denied previous use of methylenedioxymethamphetamine (ecstasy), inhalants, anabolic steroids, and injected drugs.

\section{Effects of Gender, Prenatal Exposure to Maternal Smoking, and Adolescent Smoking on Mood and on Task Performance}

We found a significant adolescent smoking by prenatal exposure interaction effect on depressed mood measured at the time of assessment $(\beta=-6.7, t=-2.1, p<0.05)$. Both adolescent smoking and prenatal exposure to maternal smoking were associated with increases in depressed mood at the time of assessment (CES-D score: prenatally exposed smokers $=11.4 \pm 8.5$, nonexposed smokers $=11.2 \pm 8.4$, prenatally exposed nonsmokers $=9.7 \pm 7.2$, nonexposed nonsmokers $=5.5 \pm 4.0$ ). This effect was most pronounced in current smokers and was not significantly modified by gender.

Consistent with previous observations (Shaywitz et al, 2001), lexical discrimination was less accurate and slower during the auditory relative to the visual simple and selective attention conditions (main effect of modality: performance accuracy: $\beta=-0.05, t=-2.2, p<0.03$; reaction time: $\beta=234.0, t=6.8, p<0.0001)$. A significant 
modality by prenatal exposure to maternal smoking by adolescent smoking effect was observed on performance accuracy $(\beta=-0.1, t=-2.0, p<0.05$; Figure $2 \mathrm{a})$. This reflected evidence of a dose-related effect of exposure, whereby prenatal or adolescent exposure alone was associated with performance accuracy that was intermediate between that of smokers with prenatal exposure, whose performance accuracy was lowest, and nonsmokers with no prenatal exposure, whose performance accuracy was highest.

This interaction effect was significantly modified by gender (modality by prenatal exposure by adolescent smoking by gender $\beta=0.1, t=2.4, p<0.02$; Figure $2 b$ ). Post hoc testing within each of the four exposure groups indicated significant effects of modality across all exposure groups, whereby lexical discrimination was less accurate during auditory than during visual conditions. This main effect of modality was significantly modified by gender only among smokers with prenatal exposure to maternal smoking (gender by modality interaction: $\beta=0.1, t=3.3$, $p<0.002$ ). Within this group, males demonstrated a greater reduction in performance accuracy during auditory conditions relative to visual conditions than females. All of these effects remained significant after controlling for the presence of residual cannabinoids (positive urine toxicology screens).

The effect of modality on reaction time was not significantly modified by prenatal exposure to maternal smoking, adolescent smoking, or gender. Although the effect of attention load on reaction time was significant $(\beta=102.4, t=3.0, p<0.004)$, reflecting slower reaction times during selective relative to simple attention conditions, effects of attention load on reaction time were not significantly modified by prenatal exposure to maternal smoking, adolescent smoking, or gender. The effect of attention load on performance accuracy was not significant. Effects of prenatal exposure to maternal smoking, adolescent smoking, and gender on performance accuracy and reaction time during the divided attention task were not significant.

Effects of Prenatal Exposure to Maternal Smoking and Adolescent Smoking on Neurocircuitry Supporting Auditory and Visual Attention

Demographic, clinical, and cognitive characteristics of the subgroup of adolescents who underwent fMRI scanning are presented in Table 2.

The groups undergoing scanning did not differ in age, gender, birth weight, symptoms of inattention, years of education, years of parent education, or alcohol consumption. Smokers had significantly more symptoms of depression at screening $(\beta=4.7, t=2.1, p<0.05)$, greater history of cannabis consumption $(\beta=211.3, t=2.3, p<0.05)$, and lower estimated general intelligence $(\beta=-8.2, t=-2.2$, $p<0.05)$. These effects of adolescent smoking were not significantly modified by gender or prenatal exposure to maternal smoking. Adolescents with prenatal exposure to maternal smoking had more environmental tobacco smoke exposure during gestation $(\beta=18.0, t=2.0, p<0.05)$. These effects of prenatal exposure were not significantly modified by gender or smoking status.
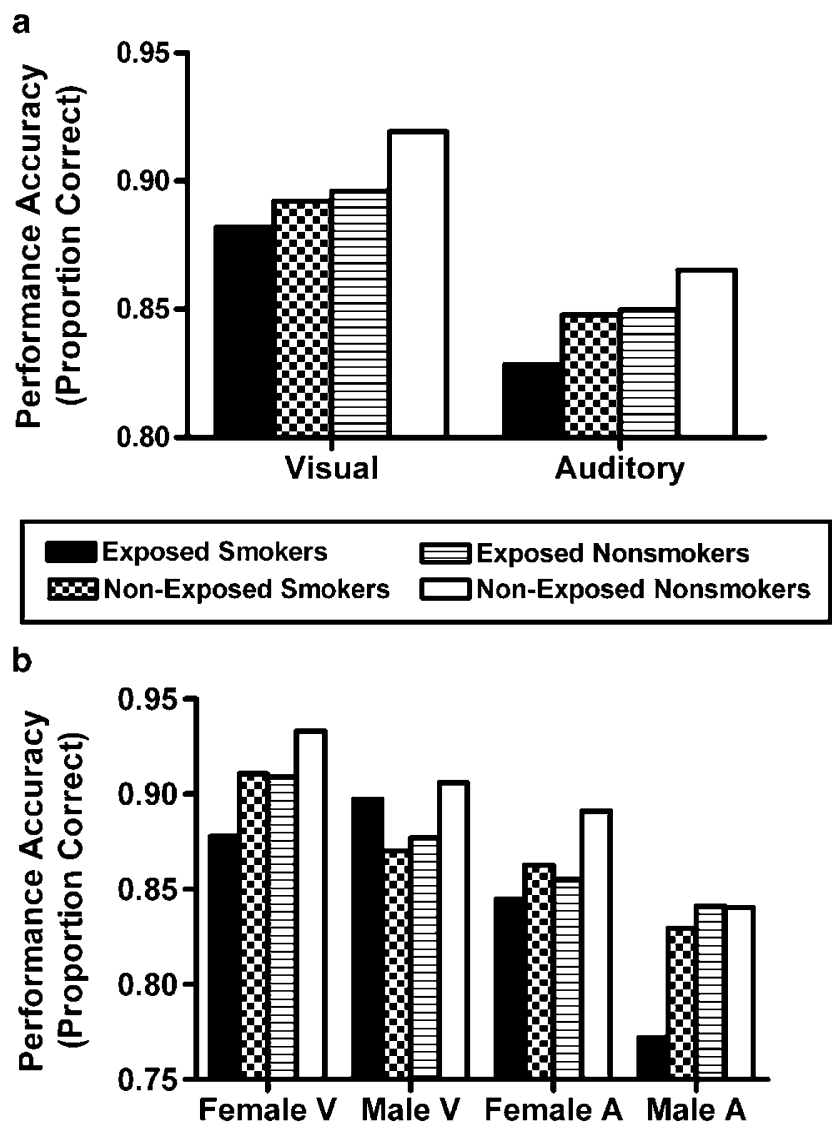

Figure 2 (a) Prenatal exposure to maternal smoking (Exposed/ Nonexposed) by adolescent smoking (Smokers/Nonsmokers) by modality interaction effect on performance accuracy during the simple/selective attention task $(\beta=-0.1, \quad t=-2.0, p<0.05)$. (b) Prenatal exposure to maternal smoking (Exposed/Nonexposed) by adolescent smoking (Smokers/Nonsmokers) by modality by gender interaction effect on performance accuracy during the simple/selective attention task $(\beta=0.1$, $t=2.4, p<0.02)$. $A=$ auditory, $V=$ visual.

Given that effects of prenatal exposure to maternal smoking and adolescent smoking on accuracy of simple/ selective attention task performance were significantly modified by modality, percent signal change data during performance of simple and selective attention tasks were extracted from regions showing significant main effects of modality. These included bilateral superior temporal gyrus (Talairach coordinates, left (BA 41/42): $X=-58, Y=-21$, $Z=12$; right (BA 42): $X=60, Y=-25, Z=7$ ), bilateral lingual gyrus (left (BA 18): $X=-13, Y=-60, Z=3$; right (BA 18): $X=25, Y=-50, Z=4$ ), and right middle frontal gyrus (BA 9: $X=31, Y=33, Z=32$ ), where activation was greater during the auditory conditions, and bilateral middle occipital gyrus (left (BA 17/18): $X=-23, Y=-89, Z=4$; right (BA 18): $X=25, Y=-89, Z=10$ ), bilateral hippocampus (left: $X=-21, Y=-22, Z=-7$; right: $X=27$, $Y=-20, Z=-9)$, and left anterior insula $(X=-33$, $Y=-5, Z=21$ ), where activation was greater during visual conditions. Percent signal change data extracted from these regions of interest were tested for significant prenatal exposure by adolescent smoking by modality interaction effects using linear mixed effects regression models, controlling for potential confounding variables including 
Table 2 Demographic, Clinical and Cognitive Characteristics of 33 Adolescents with and 30 Adolescents without Prenatal Exposure to Active Maternal Smoking Who Underwent fMRI Scanning ${ }^{a}$

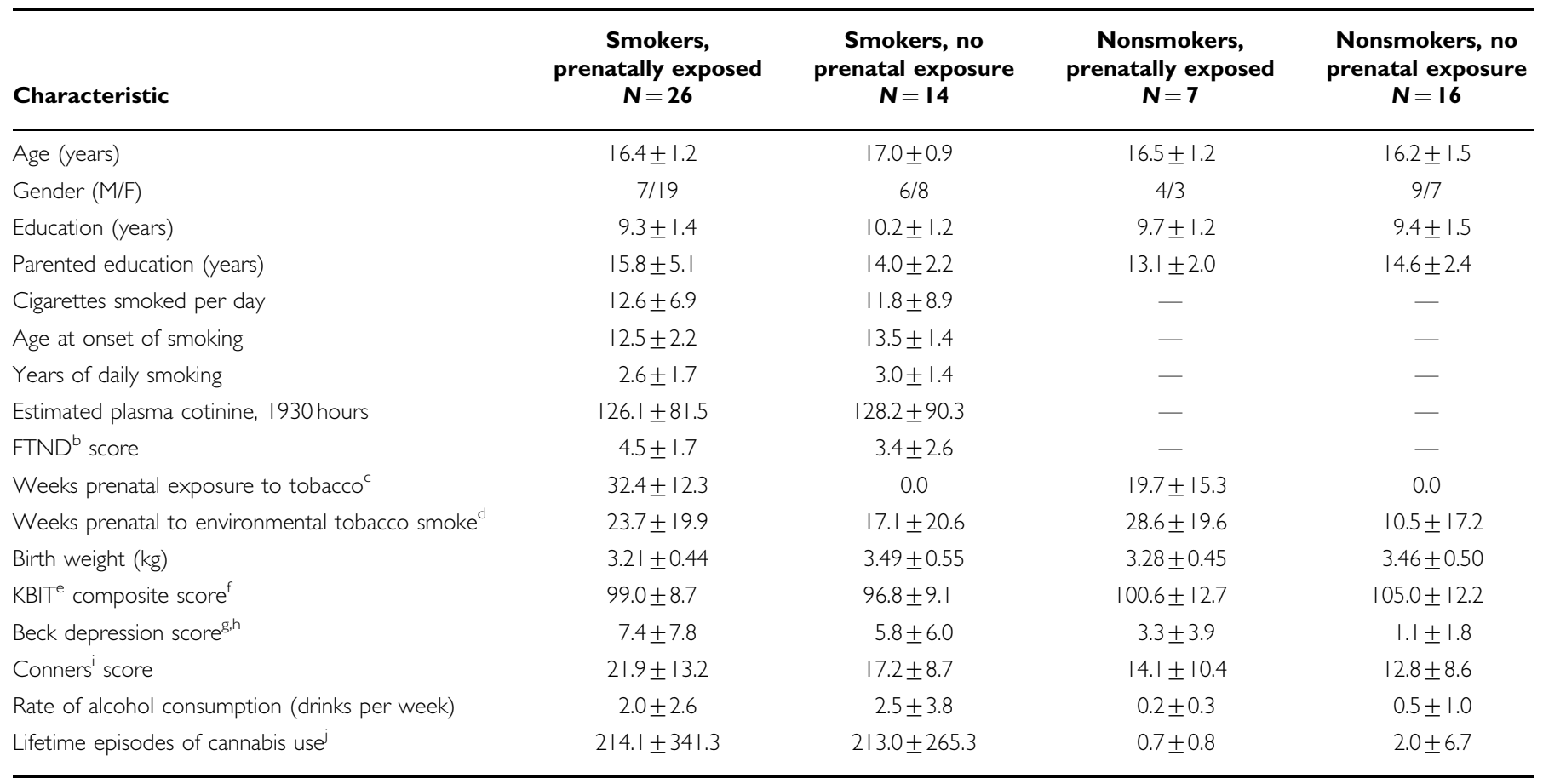

a Data are presented as mean \pm standard deviation, unless otherwise specified.

${ }^{b}$ Fagerstrom Test for Nicotine Dependence (Heatherton et al, 1991).

'Prenatally exposed $>$ no prenatal exposure: $\beta=19.7, t=4.6, p<0.00001$.

${ }^{d}$ Prenatally exposed $>$ no prenatal exposure: $\beta=18.0, t=2.0, p<0.05$.

'Kaufman Brief Intelligence Test (Bowers and Pantle, 1998).

fSmokers < nonsmokers: $\beta=-8.2, t=-2.2, p<0.05$.

'(Beck et al, 1961).

hismokers $>$ nonsmokers: $\beta=4.7, t=2.1, p<0.05$.

'Conners Adolescent Self Report Scale (Conners, 1998).

ismokers $>$ nonsmokers: $\beta=211.3, t=2.3, p<0.05$.

the presence of residual cannabinoids, as described above (see data). Effects of gender were not examined in these models given the smaller size of the sample undergoing fMRI scanning; however gender was included in the model as a control variable.

Activation of four regions showed significant prenatal exposure by adolescent smoking by modality interaction effects; bilateral superior temporal gyrus (left: $\beta=-0.2$, $t=-2.3, \quad p<0.03$; right: $\beta=-0.2, \quad t=-2.2, \quad p<0.03$; Figure 3), and bilateral lingual gyrus (left: $\beta=-0.2$, $t=-2.3, \quad p<0.03$; right: $\beta=-0.2, \quad t=-3.4, \quad p<0.002$; Figure 4). Plots of the percent signal change data revealed that, across exposure groups, activation of these regions was greater during the auditory conditions than during the visual conditions, consistent with earlier comparisons of auditory and visual attention demonstrating the importance of these regions for auditory attention (Saito et al, 2005; Shaywitz et al, 2001). Post hoc analyses indicated that during the auditory conditions, activation of right superior temporal gyrus was significantly greater in all exposure groups relative to subjects with neither prenatal nor adolescent exposure, whereas activation of left and right lingual gyrus was significantly greater in subjects with prenatal exposure to tobacco smoke alone than in subjects with neither prenatal nor adolescent exposure. Task-related activation of bilateral superior temporal and lingual gyrus in subjects with combined prenatal and adolescent exposure was not significantly different from that of subjects with adolescent or prenatal exposure to tobacco smoke alone.

\section{DISCUSSION}

In the present study, in both auditory and visual modalities, female nonsmokers with no prenatal exposure to maternal smoking performed most accurately, female smokers with prenatal exposure performed least accurately, whereas performance of females with prenatal or adolescent exposure to tobacco smoke alone was intermediate between these two extremes. This pattern suggests a linear dosedependent effect of prenatal and adolescent exposure to tobacco smoke on neurocircuitry that supports visual and auditory attention in females. In contrast, among male adolescents, deleterious effects of exposure to tobacco smoke were most pronounced among smokers with prenatal exposure during the auditory attention conditions.

Earlier work has demonstrated that intact cortical cholinergic neurotransmission is required for normal attentional performance (Sarter et al, 2005). In animals, cortical cholinergic deafferentation produces impaired 


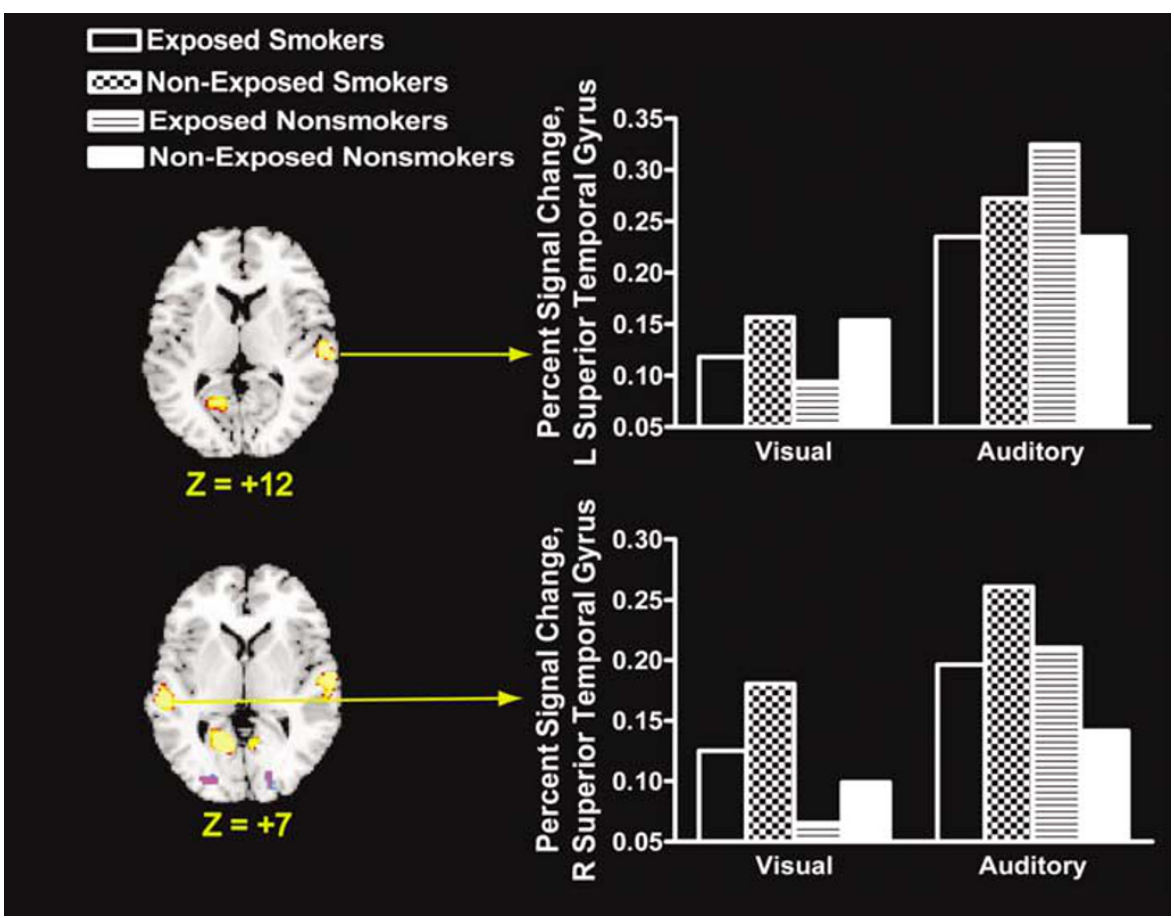

Figure 3 Left panels: prenatal exposure to maternal smoking by adolescent smoking by modality interaction effects on activation of left (BA $4 \mathrm{I} / 42$ $\beta=-0.2, t=-2.3, p<0.03$ ) and right superior temporal gyrus (BA 42: $\beta=-0.2, t=-2.2, p<0.03$ ) during performance of the simple/selective attention task. The right side of the brain is on the left side of these images. Right panels: plots of average percent signal change of left and right superior temporal gyrus for each group during auditory and visual simple/selective attention task conditions.

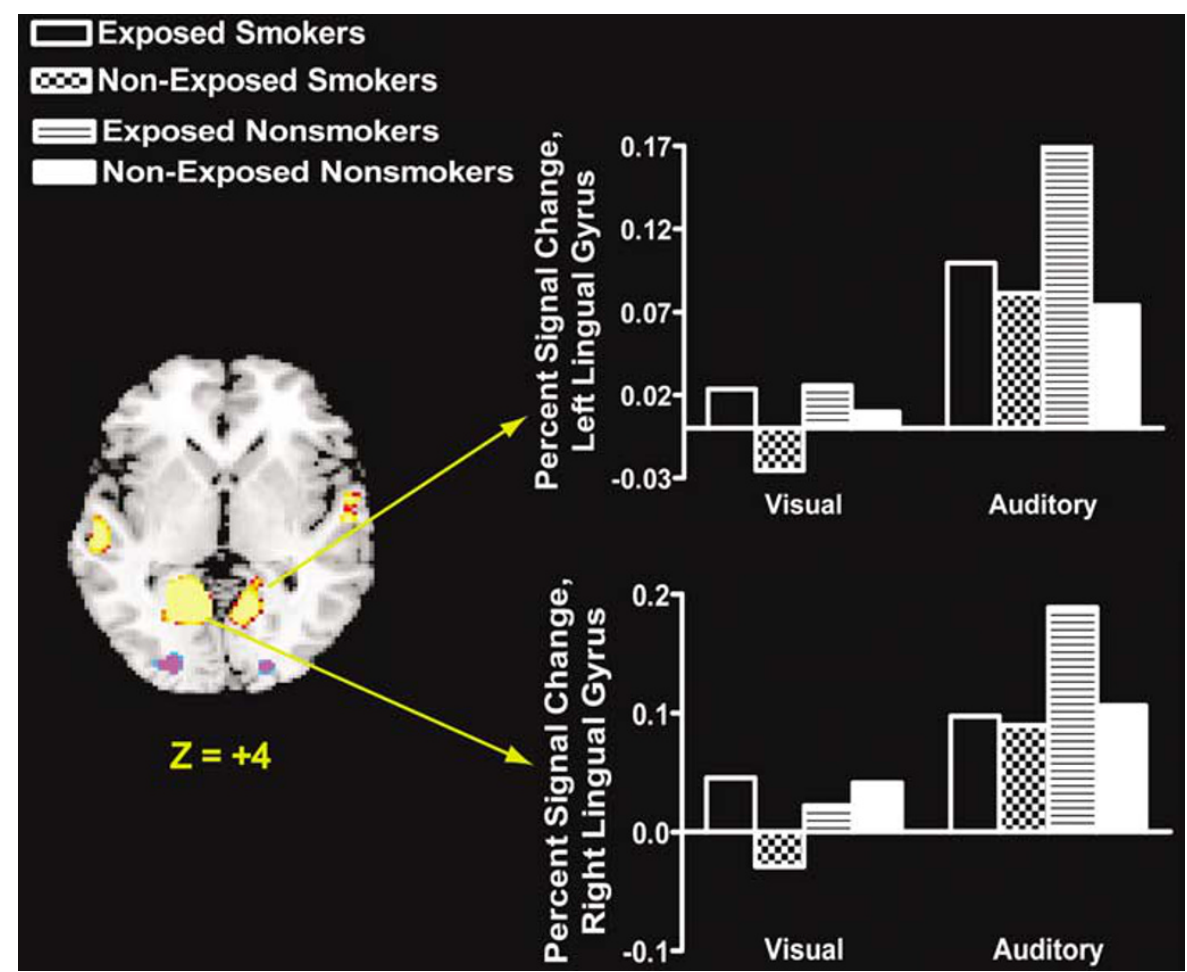

Figure 4 Left panels: prenatal exposure to maternal smoking by adolescent smoking by modality interaction effects on activation of left (BA I 8: $\beta=-0.2$, $t=-2.3, p<0.03$ ) and right lingual gyrus (BA I $8: \beta=-0.2, t=-3.4, p<0.002$ ) during performance of the simple/selective attention task. The right side of the brain is on the left side of these images. Right panels: plots of average percent signal change of left and right lingual gyrus for each group during auditory and visual simple/selective attention task conditions. 
responding to signal trials, whereas responding to nonsignal trials remains intact (McGaughy et al, 1996; McGaughy and Sarter, 1998). This suggests that cortical cholinergic hypoactivity does not alter the primary representation of sensory input, but impairs the detection process (Sarter et al, 2005). Conversely, in animals and in humans, increasing cholinergic neurotransmission has been shown to improve both visual and auditory attention (Domino and Kishimoto, 2002; Furey et al, 2000; Harkrider and Hedrick, 2005; Sarter et al, 2005). Work in a rodent model of developmental nicotine exposure has demonstrated stable reductions in markers of cortical cholinergic activity in females following prenatal or adolescent exposure to nicotine alone, with enhanced reductions in cholinergic markers following combined exposure (Slotkin et al, 2007). Together, these findings suggest that the adverse effect of prenatal and adolescent exposure to tobacco smoke on visual and auditory attention in females, which were more prominent with increasing magnitude of exposure, may reflect the impact of nicotine exposure-induced reductions in cholinergic neurotransmission, leading to impairments in attention.

In male rodents, reductions in cortical cholinergic markers following prenatal or adolescent nicotine exposure alone were observed to be similar in magnitude to those following combined exposure (Slotkin et al, 2007). Among males in the present study, a more complex, modalityspecific pattern of impairments was observed, with combined prenatal and adolescent exposure to tobacco smoke being associated with a greater reduction in performance accuracy during auditory conditions than during visual conditions. These observations suggest that in males neurocircuitry supporting auditory attention may be more vulnerable to developmental insult from nicotine than is the neurocircuitry supporting visual attention, whereas in females, neurocircuitry supporting auditory and visual attention may be equally vulnerable to the effects of nicotine exposure during prenatal and adolescent development. In turn, our findings indicate the need to return to animal models to examine brain regions involved in auditory and visual processing, to determine whether these effects are observed pursuant to developmental exposure to nicotine as opposed to tobacco smoke.

Prospective clinical studies have provided evidence that prenatal exposure to maternal smoking is associated with deficits in auditory processing among offspring that persist through adolescence (Fried et al, 1997, 2003; McCartney et al, 1994). Recent preclinical work indicates that nicotine exposure during early postnatal development, corresponding to the third trimester of pregnancy in humans, resulted in impaired function of nAChRs in primary auditory cortex localized on neurons that regulate thalamocortical inputs (Liang et al, 2006). Although gender differences in this effect of prenatal exposure alone were not noted in these studies, the present findings suggest that, in males, when prenatal exposure is followed by adolescent exposure to tobacco smoke, neurocircuitry supporting auditory attention may be more vulnerable to the developmental toxicity of nicotine than neurocircuitry supporting visual attention. In rodents, binding availability of nicotinic receptors in cortex and in subcortical structures does not differ by sex (Slotkin et al, 2007). Thus, the gender-specific effect of developmental exposure to tobacco smoke on auditory attention may not stem from global male-female differences in nicotinic receptor-binding sites, but rather to gender differences in downstream effects of activation of nAChRs by nicotine or in the hormonal regulation of these downstream effects. Alternatively, sex differences in receptor expression or function may emerge with finer dissection of subregions that are specifically involved in auditory tasks, and future work needs to address this issue.

Consistent with the notion that in humans, neurocircuitry supporting auditory attention may be more vulnerable to the developmental toxicity of nicotine than neurocircuitry supporting visual attention, functional-imaging data revealed significant prenatal exposure by adolescent smoking by modality effects in regions that support auditory attention, including bilateral superior temporal gyrus, encompassing primary auditory cortex on the left (Saito et al, 2005; Shaywitz et al, 2001). During performance of the auditory attention tasks, activation of right superior temporal gyrus was greater in all exposure groups relative to subjects with neither prenatal nor adolescent exposure, whereas activation of bilateral lingual gyrus was greater in subjects with prenatal exposure to tobacco smoke alone relative to subjects with no exposure. These data suggest a loss of efficiency in cortical regions that support auditory attention, possibly stemming from reduced cortical cholinergic neurotransmission resulting from exposure to tobacco smoke during prenatal or adolescent brain development (Abreu-Villaca et al, 2003a, b; Slotkin et al, 2007). Earlier work in humans and animals has shown that enhanced cholinergic neurotransmission improves the selectivity of perceptual processing (Furey et al, 2000; McGaughy et al, 1996; McGaughy and Sarter, 1998; Sarter et al, 2005). In humans, this effect is associated with reduced activation of regions mediating higher cortical processing (Furey et al, 2000). Together, these observations suggest that reductions in cortical cholinergic neurotransmission resulting from exposure to tobacco smoke during prenatal or adolescent brain development may reduce the efficiency of central auditory processing by reducing the selectivity of auditory perception.

Earlier studies have shown that prenatal exposure to active maternal smoking increases risk for deficits in auditory processing (Fried et al, 1997; McCartney et al, 1994) and Attention Deficit Hyperactivity Disorder (ADHD) (Linnet et al, 2003; Romano et al, 2006; Williams et al, 1998) among offspring. In turn, ADHD symptoms have been shown to increase risk for tobacco smoking in a linear, dose-dependent manner (Kollins et al, 2005). Male gender is an independent risk factor for ADHD (Romano et al, 2006), and many of these studies have controlled for effects of gender (Linnet et al, 2003; Romano et al, 2006; Williams et al, 1998). However, few have tested whether risk of developing ADHD symptoms subsequent to prenatal exposure to maternal smoking is modified by gender. Shafritz and colleagues (2004), using the task described herein in a predominantly $(73 \%)$ male sample, observed significant reductions in accuracy during performance of auditory simple and auditory selective attention but not visual simple or visual selective attention tasks among subjects with ADHD (Shafritz et al, 2004). fMRI scanning revealed altered function of bilateral middle temporal gyrus 
during performance of the auditory attention conditions but not during performance of the visual attention conditions in unmedicated ADHD patients relative to healthy comparison subjects (Shafritz et al, 2004). Neither smoking status nor prenatal history was described in this sample.

In the present study, both adolescent smoking and prenatal exposure to maternal smoking were associated with increases in symptoms of depressed mood. Earlier work has demonstrated alterations in serotonin receptor $1 \mathrm{~A}$ $\left(5 \mathrm{HT}_{1 \mathrm{~A}}\right)$ and $2\left(5 \mathrm{HT}_{2}\right)$ binding in patients with depression (Arango et al, 2001; Parsey et al, 2006; Yatham et al, 2000). In the rodent model, both prenatal and adolescent exposure to nicotine were found to increase cerebrocortical $5 \mathrm{HT}_{1 \mathrm{~A}}$ and $5 \mathrm{HT}_{2}$ receptor binding, while shifting $5 \mathrm{HT}$ signaling patterns toward inhibition (Slotkin et al, 2006, 2007; Xu et al, 2002), suggesting that tobacco exposure-associated increases in depressed mood observed in the present sample may stem from nicotine-induced alterations in 5HT receptor function. Although effects of prenatal and adolescent nicotine exposure on $5 \mathrm{HT}_{1 \mathrm{~A}}$ and $5 \mathrm{HT}_{2}$ receptor binding were greater in males in the rodent model (Slotkin et al, 2007), gender differences in the impact of prenatal or adolescent exposure to tobacco smoke on depressed mood in human adolescents did not achieve statistical significance in the present study.

The possibility that the group differences in attentional performance and cortical processing efficiency observed in the present study stem from factors unrelated to prenatal or adolescent exposure to tobacco smoke or gender cannot be excluded. However, the fact that interaction effects remained significant after group matching and statistical controls for potentially confounding variables, including cannabis and alcohol use and maternal use of alcohol, argues against this possibility, as does the congruence of many of our conclusions with the findings from rodent models of exposure to nicotine alone. Similarly, although prenatal exposure to maternal smoking was associated with a small reduction in birth weight, the lack of a significant effect of prenatal exposure on estimated IQ argues against a nonspecific fetal insult causing the pattern of deficits observed in the present study (Slotkin, 2004). Although the gender distribution within exposure groups was uneven, the analyses performed produce unbiased estimates of the population means regardless of the sample size, and take into account the increased variability of estimates derived from smaller samples when computing $p$ values. The size of the sample undergoing fMRI scanning in the present study did not permit evaluation of the degree to which gender modifies the effect of prenatal and adolescent exposure to tobacco smoke on the function of neurocircuitry supporting visual and auditory attention. Imaging of this sample is ongoing and future work will examine these issues. Other limitations include the measurement of prenatal exposure by retrospective self-report. Work comparing prospectively and retrospectively collected data about pregnancy has supported the accuracy of pregnancy data collected by retrospective self-report (Buka et al, 2004; Jacobson et al, 1991). Similarly, comparison of serum cotinine concentrations and self-reported smoking behavior during pregnancy has yielded evidence of significant agreement between these measures (Buka et al, 2003). Although the lower birth weight observed in prenatally exposed subjects is consistent with many earlier studies linking maternal smoking during pregnancy with lower birth weight (Eskenazi et al, 1995) and thus further supports the validity of our retrospectively acquired data, rates of smoking during pregnancy may have been underreported in the present study owing to the social stigma attached to this behavior. Finally, the possibility that genetic factors shared by mothers and their offspring may mediate both increased risk for smoking and impaired visual and auditory attention cannot be excluded by these data.

In summary, the present findings suggest that in females, exposure to tobacco smoke during prenatal and adolescent developmental epochs exerts deleterious effects on auditory and visual attention that increase with increasing magnitude of exposure. These data are consistent with observations in the female rodent model of prenatal and adolescent nicotine exposure and suggest that observed attentional impairments may stem from nicotine exposure-induced reductions in cholinergic neurotransmission (Slotkin et al, 2007). Although the neurocircuitry supporting auditory and visual attention appears to be equally vulnerable to developmental insult stemming from exposure to tobacco smoke in females, in males, neurocircuitry supporting auditory attention appears to be more vulnerable to this insult than is neurocircuitry supporting visual attention. Prenatal exposure to maternal smoking was associated with reduced efficiency of neurocircuitry supporting auditory attention, consistent with reduced cortical cholinergic neurotransmission. Recent data indicate that between 10 and $16 \%$ of women who give birth in the United States are unable to stop smoking during their pregnancies (Martin et al, 2006). The present findings underscore the importance of developing smoking prevention programs that target women of childbearing age and of developing effective treatments for tobacco dependence that do not involve nicotine replacement. Further, these data suggest the importance of assessing prenatal and adolescent exposure to tobacco smoke in studies of cognition and brain function in clinical populations with attentional impairments.

\section{ACKNOWLEDGEMENTS}

This research was supported by National Institutes of Health grants DA14655, DA017333, and RR06022. We thank Jonathan S Feinstein for statistical consultation, $\mathrm{R}$ Todd Constable for consultation regarding image acquisition, Irina $\mathrm{N}$ Krasonova and Jean Lud Cadet for critical readings of this manuscript, and the subjects and their families for their participation in this study.

\section{DISCLOSURE/CONFLICTS OF INTEREST}

Theodore A Slotkin has served as an expert witness on behalf of governmental entities, corporations and/or individuals. These activities were unrelated to the work described herein and represent no actual or apparent conflict of interest.

\section{REFERENCES}

Abreu-Villaca Y, Seidler FJ, Qiao D, Tate CA, Cousins MM, Thillai I et al (2003a). Short-term adolescent nicotine exposure has 
immediate and persistent effects on cholinergic systems: critical periods, patterns of exposure, dose thresholds. Neuropsychopharmacology 28: 1935-1949.

Abreu-Villaca Y, Seidler FJ, Tate CA, Slotkin TA (2003b). Nicotine is a neurotoxin in the adolescent brain: critical periods, patterns of exposure, regional selectivity, and dose thresholds for macromolecular alterations. Brain Res 979: 114-128.

American Psychiatric Association (1994). Diagnostic and Statistical Manual of Mental Disorders, Fourth Edition (DSM-IV). American Psychiatric Association: Washington DC.

Arango V, Underwood MD, Boldrini M, Tamir H, Kassir SA, Hsiung $S$ et al (2001). Serotonin 1A receptors, serotonin transporter binding and serotonin transporter mRNA expression in the brainstem of depressed suicide victims. Neuropsychopharmacology 25: 892-903.

Ashburner J, Friston KJ (1999). Nonlinear spatial normalization using basis functions. Hum Brain Mapp 7: 254-266.

Beck AT, Ward CH, Mendelson M, Mock J, Erbaugh J (1961). An inventory for measuring depression. Arch Gen Psychiatry 4: 561-571.

Benes FM, Turtle M, Khan Y, Farol P (1994). Myelination of a key relay zone in the hippocampal formation occurs in the human brain during childhood, adolescence, and adulthood. Arch Gen Psychiatry 51: 477-484.

Bowers TL, Pantle ML (1998). Shipley institute for living scale and the Kaufman Brief Intelligence Test as screening instruments for intelligence. Assessment 5: 187-195.

Buka SL, Goldstein JM, Spartos E, Tsuang MT (2004). The retrospective measurement of prenatal and perinatal events: accuracy of maternal recall. Schizophr Res 71: 417-426.

Buka SL, Shenassa ED, Niaura R (2003). Elevated risk of tobacco dependence among offspring of mothers who smoked during pregnancy: a 30-year prospective study. Am J Psychiatry 160: $1978-1984$.

Conners CK (1998). Rating scales in attention-deficit/hyperactivity disorder: use in assessment and treatment monitoring. J Clin Psychiatry 59(Suppl 7): 24-30.

Cornelius MD, Leech SL, Goldschmidt L, Day N (2000). Prenatal tobacco exposure: is it a risk factor for early tobacco experimentation? Nicotine Tob Res 2: 45-52.

Domino EF, Kishimoto T (2002). Tobacco smoking increases gating of irrelevant and enhances attention to relevant cues. Nicotine Tob Res 4: 71-78.

Eskenazi B, Prehn AW, Christianson RE (1995). Passive and active maternal smoking as measured by serum cotinine: the effect on birthweight. Am J Public Health 85: 395-398.

Fried PA, Watkinson B, Gray R (2003). Differential effects on cognitive functioning in 13- to 16-year-olds prenatally exposed to cigarettes and marihuana. Neurotoxicol Teratol 25: 427-436.

Fried PA, Watkinson B, Gray R (2006). Neurocognitive consequences of cigarette smoking in young adults - a comparison with pre-drug performance. Neurotoxicol Teratol 28: 517-525.

Fried PA, Watkinson B, Siegel LS (1997). Reading and language in 9- to 12-year olds prenatally exposed to cigarettes and marijuana. Neurotoxicol Teratol 19: 171-183.

Furey ML, Pietrini P, Haxby JV (2000). Cholinergic enhancement and increased selectivity of perceptual processing during working memory. Science 290: 2315-2319.

Genovese CR, Lazar NA, Nichols T (2002). Thresholding of statistical maps in functional neuroimaging using the false discovery rate. Neuroimage 15: $870-878$.

Giedd JN, Clasen LS, Lenroot R, Greenstein D, Wallace GL, Ordaz S et al (2006). Puberty-related influences on brain development. Mol Cell Endocrinol 254-255: 154-162.

Griesler PC, Kandel DB, Davies M (1998). Maternal smoking in pregnancy, child behavior problems, and adolescent smoking. J Res Adolesc 8: 159-185.
Harkrider AW, Hedrick MS (2005). Acute effect of nicotine on auditory gating in smokers and non-smokers. Hear Res 202: $114-128$.

Heatherton TF, Kozlowski LT, Frecker RC, Fagerstrom KO (1991). The Fagerstrom test for nicotine dependence: a revision of the Fagerstrom Tolerance Questionnaire. Br J Addict 86: 1119-1127.

Hughes J, Hatsukami DK (1998). Errors in using tobacco withdrawal scale. Tob Control 7: 92-93.

Huttenlocher PR (1979). Synaptic density in human frontal cortex: developmental changes and effects of aging. Brain Res 163: 195-205.

Jacob P, Wilson M, Benowitz NL (1981). Improved gas chromatographic method for the determination of nicotine and cotinine in biologic fluids. J Chromatogr 222: 61-70.

Jacobsen LK, Krystal JH, Mencl WE, Westerveld M, Frost SJ, Pugh KR (2005). Effects of smoking and smoking abstinence on cognition in adolescent tobacco smokers. Biol Psychiatry 57: q56-66.

Jacobson SW, Jacobson JL, Sokol RJ, Martier SS, Ager JW, Kaplan MG (1991). Maternal recall of alcohol, cocaine, and marijuana use during pregnancy. Neurotoxicol Teratol 13: 535-540.

Jarvis MJ, Primatesta P, Erens B, Feyerabend C, Bryant A (2003). Measuring nicotine intake in population surveys: comparability of saliva cotinine and plasma cotinine estimates. NicotineTob Res 5: 349-355.

Kandel DB, Wu P, Davies P (1994). Maternal smoking during pregnancy and smoking by adolescent daughters. Am J Public Health 84: 1407-1413.

Kaufman J, Birmaher B, Brent D, Rao U, Flynn C, Moreci P et al (1997). Schedule for affective disorders and schizophrenia for school-age children-present and lifetime version (K-SADS-PL): initial reliability and validity data. J Am Acad Child Adolesc Psychiatry 36: 980-988.

Kollins SH, McClernon FJ, Fuemmeler BF (2005). Association between smoking and attention-deficit/hyperactivity disorder symptoms in a population-based sample of young adults. Arch Gen Psychiatry 62: 1142-1147.

Lauder JM, Schambra UB (1999). Morphogenetic roles of acetylcholine. Environ Health Persp 107(Suppl 1): 65-69.

Lenroot RK, Giedd JN (2006). Brain development in children and adolescents: insights from anatomical magnetic resonance imaging. Neuroscience Biobehav Rev 30: 718-729.

Liang K, Poytress BS, Chen Y, Leslie FM, Weinberger NM, Metherate R (2006). Neonatal nicotine exposure impairs nicotine enhancement of central auditory processing and auditory learning in adult rats. Eur J Neurosci 24: 857-866.

Linnet KM, Dalsgaard S, Obel C, Wisborg K, Henriksen TB, Rodriguez A et al (2003). Maternal lifestyle factors in pregnancy risk of attention deficit hyperactivity disorder and associated behaviors: review of the current evidence. Am J Psychiatry 160: 1028-1040.

Martin JA, Hamilton BE, Sutton PD, Ventura SJ, Menacker F, Kirmeyer S (2006). Births: Final Data for 2004. Natl Vital Stat Rep 55: 1-102.

McCartney JS, Fried PA, Watkinson B (1994). Central auditory processing in school-age children prenatally exposed to cigarette smoke. Neurotoxicol Teratol 16: 269-276.

McGaughy J, Kaiser T, Sarter M (1996). Behavioral vigilance following infusions of 192 IgG-saporin into the basal forebrain: selectivity of the behavioral impairment and relation to cortical AChE-positive fiber density. Behav Neurosci 110: 247-265.

McGaughy J, Sarter M (1998). Sustained attention performance in rats with intracortical infusions of 192 IgG-saporin-induced cortical cholinergic deafferentation: effects of physostigmine and FG 7142. Behav Neurosci 112: 1519-1525.

Metherate R, Hsieh CY (2004). Synaptic mechanisms and cholinergic regulation in auditory cortex. Prog Brain Res 145: $143-156$ 
Meyers K, McLellan AT, Jaeger JL, Pettinati HM (1995). The development of the comprehensive addiction severity index for adolescents (CASI-A). An interview for assessing multiple problems of adolescents. J Subst Abuse Treat 12: 181-193.

Navarro HA, Seidler FJ, Eylers JP, Baker FE, Dobbins SS, Lappi SE et al (1989). Effects of prenatal nicotine exposure on development of central and peripheral cholinergic neurotransmitter systems. Evidence for cholinergic trophic influences in developing brain. J Pharmacol Exp Ther 251: 894-900.

Papademetris X, Jackowski AP, Schultz RT, Staib LH, Duncan JS (2003). Computing 3D non-rigid brain registrations using extended robust point matching for composite multisubject fMRI analysis. In: Ellis RE, Peters TM (eds). Medical Image Computing and Computer Assisted Intervention. SpringerVerlag: Berlin. pp 788-795.

Parsey RV, Oquendo MA, Ogden RT, Olvet DM, Simpson N, Huang YY et al (2006). Altered serotoning 1A binding in major depression: a [carbonyl-C-11]WAY100635 positron emission tomography study. Biol Psychiatry 59: 106-113.

Peachey JE, Lei H (1988). Assessment of opioid dependence with naloxone. Br J Addict 83: 193-201.

Pineda JA, Herrera C, Kang C, Sandler A (1998). Effects of cigarette smoking and 12-h abstention on working memory during a serial-probe recognition task. Psychopharmacology 139: 311-321.

Radloff L (1977). The CES-D scale; a self report depression scale for research in the general population. Appl Psychol Measures 1: 385-401.

Romano E, Tremblay RE, Farhat A, Cote S (2006). Development and prediction of hyperactive symptoms from 2 to 7 years in a population-based sample. Pediatrics 117: 2101-2109.

Saito DN, Yoshimura K, Kochiyama T, Okada T, Honda M, Sadato $\mathrm{N}$ (2005). Cross-modal binding and activated attentional networks during audio-visual speech integration: a functional MRI study. Cereb Cortex 15: 1750-1760.

Sarter M, Hasselmo ME, Bruno JP, Givens B (2005). Unraveling the attentional functions of cortical cholinergic inputs: interactions between signal-driving and cognitive modulation of signal detection. Brain Res Rev 48: 98-111.

Shafritz KM, Marchione KE, Gore JC, Shaywitz SE, Shaywitz BA (2004). The effects of methylphenidate on neural systems of attention in attention deficit hyperactivity disorder. Am J Psychiatry 161: 1990-1997.

Shaywitz BA, Shaywitz SE, Pugh KR, Fulbright RK, Skudlarski P, Mencl WE et al (2001). The functional neural architecture of components of attention in language-processing tasks. Neuroimage 13: 601-612.
Shiffman S, Paty JA, Gnys M, Kassel JD, Elash C (1995). Nicotine withdrawal in chippers and regular smokers: subjective and cognitive effects. Health Psychol 14: 301-309.

Slotkin TA (2004). Cholinergic systems in brain development and disruption by neurotoxicants: nicotine, environmental tobacco smoke, organophosphates. Toxicology Appl Pharmacol 198: $132-151$.

Slotkin TA, MacKillop EA, Rudder CL, Ryde IT, Tate CA, Seidler FJ (2007). Permanent, sex-selective effects of prenatal or adolescent nicotine exposure, separately or sequentially, in rat brain regions: indices of cholinergic and serotonergic synaptic function, cell signaling, and neural cell number and size at 6 months of age. Neuropsychopharmacology doi:10.1038/ sj.npp.1301231.

Slotkin TA, Tate CA, Cousins MM, Seidler FJ (2006). Prenatal nicotine exposure alters the responses to subsequent nicotine administration and withdrawal in adolescence: serotonin receptors and cell signaling. Neuropsychopharmacology 31: 2462-2475.

Snyder FR, Davis FC, Henningfield JE (1989). The tobacco withdrawal syndrome: performance decrements assessed on a computerized test battery. Drug Alcohol Depend 23: 259-266.

Sobell LC, Sobell MB (1992). Timeline follow-back: a technique for assessing self-reported alcohol consumption. In: Litten R, Allen J (eds). Measuring Alcohol Consumption. The Humana Press: Totowa, NJ.

Talairach J, Tournoux P (1988). Co-Planar Stereotaxic Atlas of the Human Brain. Thieme Medical: New York.

West R, Hack S (1991). Effect of cigarettes on memory search and subjective ratings. Pharmacol Biochem Behav 38: 281-286.

Williams GM, O'Callaghan M, Najman JM, Bor W, Andersen MJ, Richards D et al (1998). Maternal cigarette smoking and child psychiatric morbidity: a longitudinal study. Pediatrics 102: 1-8.

Woods RP (1996). Modeling for intergroup comparisons of imaging data. Neuroimage 4: S84-S94.

Xu Z, Seidler FJ, Cousins MM, Slikker W, Slotkin TA (2002). Adolescent nicotine administration alters serotonin receptors and cell signaling mediated through adenylyl cyclase. Brain Res 951: 280-292.

Yakovlev PI, Lecours AR (1967). The myelogenetic cycles of regional maturation of the brain. In: Minkowski A (ed). Regional Development of the Brain in Early Life. Blackwell Scientific: Oxford. pp 3-70.

Yatham LN, Liddle PF, Shiah IS, Scarrow G, Lam RW, Adam MJ et al (2000). Brain sertonin2 receptors in major depression: a positron emission tomography study. Arch Gen Psychiatry 57: 850-858. 